\title{
STUDY IN REDUCTION OF VORTEX DRAG AT LOW CRUISING AIRCRAFT SPEEDS
}

\author{
Mohamed B.W. Nabhan ${ }^{1 *}$, Ebrahim K. Seddiq ${ }^{2}$, \\ *1,2 University of Bahrain, College of Engineering, Mechanical Engineering Department, PO Box 32038, Isa Town, \\ Kingdom of Bahrain, Tel.+973-17876215, Fax+973-1787681234 Email: mnabhan@uob.edu.bh
}

*Corresponding Author: -

Email:mnabhan@uob.edu.bh

\begin{abstract}
: -
Aerodynamic characteristics of plain wing designed for Light Sport Aircraft has been studied. The fluid characteristics include induced drag and lift to drag ratio. Then, winglets are added to reduce the induced drag and increase the lift to drag ratio which are affected by the wing tip vortices. The theoretical, and numerical approaches are used to verify the results. A rectangular untwisted $9.528 \mathrm{~m}$ wing spans with an Airfoil NACA 4412 was used for the basic design. Winglets are added with a tip airfoil of NACA 0012, side angle of $65^{\circ}$ and new projected area of $10.328 \mathrm{~m}^{2}$. Lift and drag coefficients are used as means to measure the improvement of the aerodynamic characteristics. The wing tip vortices increase the induced drag and spoil the lift over the wing's surface. The winglets design main objectives are to decrease the induced drag, decrease fuel consumption, and increase flight safety, especially in take-off condition.

The wing with winglets model was simulated first using 3-D Fluent ANSYS version 14 at $50 \mathrm{~m} / \mathrm{s}$ velocity and $\left(0^{\circ}\right.$, $5^{\circ}$, and $10^{\circ}$ ) angles of attack with laminar flow and standard atmospheric conditions at $15^{\circ} \mathrm{C}$, and $101 \mathrm{kPa}$ and all other flow parameters as well. The second verification method was to simulate the 3-D model using the 3-D Foil Multi-Surfaces code again with the same flow parameters. Finally, the last verification method was to solve the problem theoretically using the theoretical governing equations. The theoretical solutions were used as a base line for all other results. The total drag reduction observed from the calculations with winglets is about $7.4 \%$ during the takeoff regime using theoretical calculations, where the induced drag contributes about $77 \%$ of total drag of the plain wings. The lift to drag ratio improved also in our designed model wing with winglets by $14 \%$ from the plain wing design.
\end{abstract}

Keywords: Induced drag, low flying aircraft, winglets, CFD.

\section{(우 $\circledast$ (i)}




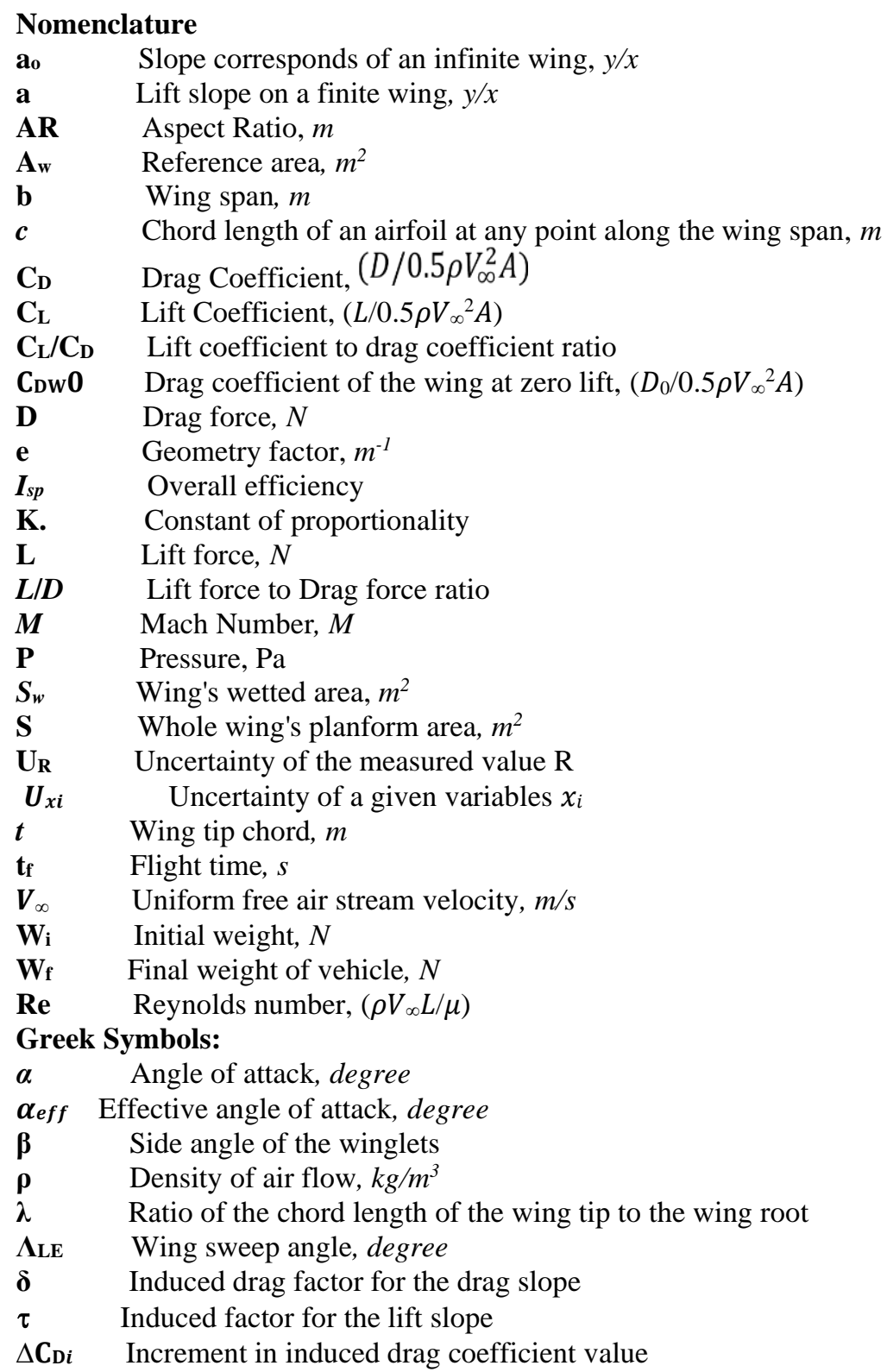

\section{INTRODUCTION}

Winglets belong to the class of wingtip devices aimed to reduce induced drag. Selection of the wingtip device depends on the specific situation and the airplane model. In the case of winglets, the reduction of the induced drag is accomplished by acting like a small sail whose lift component generates a traction force, draining energy from the tip vortices (Bento Mattos, Antonini Macedo, \& Durval Silva Filho, 2003) [1]. Winglets are a type of wing tip devices, which are used to enhance aerodynamic efficiency of wings. In general, a winglet produces a side force, which has a forward component because of the side wash produced by the wing tip circulation. This forward component of the lift acts as a thrust force reducing the aircraft induced drag, which is about $40 \%$ of the total drag in cruise and $80-90 \%$ of the total drag in the second climb segment (Kroo, 2005) [2]. A research tried to optimize winglet for minimum drag, while defining some constraints to avoid a huge weight penalty (Hantrais-Gervois et al., 2009) [3]. These constraints usually limit the wing root bending moment. However, using root bending moment is not a good indicator for the wings total weight, because it does not account for the effect of chord wise changes in the platform on the bending weight. Some researchers used low fidelity semi-empirical methods (Ning \& Kroo, 2010) [4] or high fidelity finite element analysis (Jansen, Perez, \& Ra Martins, 2010) [5] for wing weight estimation. In this research, a medium level of fidelity aerodynamic solver together with a medium level of fidelity weight estimation tool is used for winglet analysis. Using a medium level of fidelity allows reducing the computational time comparing to higher level of fidelity tools while keeping the level of accuracy high enough. Drag is caused by the induced drag that is created by the downwash of airflow onto the upper surface of the wing at the wingtips thus producing vortices that trail in the aircraft's wake. The most important wingtip devise's functions are reducing the induced drag and consequently, the trailing vortex strength. By minimizing the induced drag, and thus the wingtip vortices produced by an aircraft's wing, the energy required to create the tip vortices can be conserved and the total drag on the wing reduced (Yahaya \& Sheriff, 2012) [6]. A research by NASA Langley research center developed the concept of winglet technology. According to Gimmestad, winglet could be described as the small wing like vertical structures which extends from the wingtip, aiming at reduction in induced drag when compared to other wing tip devices or extensions. He also claimed in his research that the winglet showed $20 \%$ reduction in induced drag when compared to 
tip extension and also improved lift-to-drag ratio. Unfortunately, his theory could not reduce the overall drag of aircraft despite reducing the induced drag. The increase in the viscous drag during cruise conditions outruns the reduction in induced drag (Gimmestad, 1981) [7]. Computational Fluid Dynamics (CFD) has been used by various researchers for studying winglet performance, as it provides a reliable solution for modeling viscous effects as well. For example, Azlin et al. (Azlin, Taib, Kasolang, \& Muhammad, 2011) [8] examined the performance of winglets at low subsonic flow. They studied two types of design, elliptical and semi-circular winglets with different angles, attached on a wing at various angles of attack. Takenaka et al. also used CFD in order to perform a multidisciplinary design exploration technique regarding winglets for a commercial aircraft (Takenaka, Hatanaka, Yamazaki, \& Nakahashi, 2008) [9].

The aim of this work is to study the aerodynamic characteristics in low subsonic speeds (incompressible) laminar/ turbulent flow over airfoil NACA 4412 and straight rectangular wing which are designated to an Australian Light Sport Aircraft developed as touring/training aircraft and manufactured by Jabiru Aircraft Company. The renowned aircraft company (Jabiru) manufactures a light training aircraft without winglets installed on the wings.

\section{Plain Wing Theory}

Fig. 1 represents a view of a wing plan form with some of the important dimensions, angles and parameters used to describe the shape of an aircraft wing. The wing span, $b$, is measured from wing tip to the other wing tip. The symbol $c$ is used for the chord length of an airfoil at any point along the wing span. The subscript $r$ indicates the chord length at the wing root or the aircraft centerline. The subscript $t$ denotes the wing tip chord. In our design, $\mathrm{r}=t$ because of the rectangular wing shape. The overbar, $c$ denotes an average value of chord length for the entire wing. $\lambda$ then can be defined as the ratio of the chord length of the wing tip to the wing root, which equals to one in our project. The symbol $A R$ is used for a parameter called aspect ratio. Aspect ratio indicates the effective area which is proposed to produce lift. The symbol $\Lambda_{\mathrm{LE}}$ is used for wing sweep angle with the subscript $L E$ denoting the wing leading edge. In our design this angle equals $0^{\circ}$ because our wing is rectangular. The subscript 25 denotes the line connecting the $25 \%$ chord positions on each airfoil of the wing. The wing understudy has a sweep angle of zero degrees, thus no geometric twist occurs in the wing span.

\subsection{Wingtip vortices}

The flow around a wing section which spans the test section of a wind tunnel approximates the flow around a wing with an infinite span, no twist, and a constant chord length along its span. In this article, this type of flow was labeled twodimensional, because flow properties did not vary in the $\mathrm{z}$ direction. The flow field around a finite wing or wing with a finite span is not two-dimensional. The majority of differences between the flow around a finite wing and that around an infinite wing result from flow phenomena which occur at the wingtips. Fig. 2 represents a front view of the flow field around a finite wing. Note that the differences between the pressures above and below the wing which produce lift also produce a strong flow around the wing tip. The arrows are intended to show a front view of flow streamlines in the plane of the $50 \%$ chord point on the wing.

As shown in Fig.3, these circular flow patterns around the wing tips become concentrated into very strong tornado-like swirling flows known as wingtip vortices or trailing vortices. The circular flow pattern around the wingtips results in a downward component to the flow over the wing known as Downwash.

The change in flow direction due to downwash is called the downwash angle, and is given the symbol $\square$. The angle between the airfoil chord line and the local flow velocity vector is called the effective angle of attack, $\alpha_{\text {eff. }}$ Each individual wing section's lift, drag, and angle of attack vary with the airfoil's orientation to this local flow direction, but the whole wing's lift, drag and angle of attack must still be defined relative to the free stream direction. Fig. 4 reveals that, as a consequence of the change in effective flow direction caused by the downwash, the effective angle of attack of the airfoil is reduced, and the lift generated by each airfoil has a component in the wing's drag direction. This component of lift in the drag direction is called induced drag. The reduction in effective angle of attack due to the downwash causes the wing to produce less lift than it would if there were no downwash.

The lift, drag coefficients for an airfoil are given by the following relations (J. D. Anderson, 2003);

$$
\begin{gathered}
C_{L}=L / q S \\
C_{D}=D / q S
\end{gathered}
$$

The reduction in effective angle of attack due to downwash decreases lift at any given $\alpha_{e f f}$ and delays stall to higher values of $\alpha$.

Fig.4 represents the induced drag as a component of the three-dimensional lift in the drag direction:

$$
D_{i}=L \sin \varepsilon
$$

or $C_{D i}=C_{L} \sin \varepsilon$

It can be shown that the induced angle of attack (downwash angle, $\varepsilon$ ) along the span of wings with elliptical lift distributions is given by (J. D. Anderson, 2003);

$$
\varepsilon=\frac{C_{L}}{\pi A R} r a d \quad \text { and } \quad \mathrm{AR}=\frac{S^{2}}{\mathrm{~A}}
$$

The summation of parasite and induced drag results in the total drag coefficient;

$$
C_{D}=C_{d}+C_{D i}
$$

The induced drag coefficient $\mathrm{C}_{\mathrm{Di}}$ is proportional to square of the lift coefficient $\mathrm{C}_{\mathrm{L}}$, and inversely to the aspect ratio $\mathrm{AR}$ and the shape of the wing e. The relation between the induced 
drag coefficient and the lift coefficient is for $\varepsilon$ small, $\sin \varepsilon \approx \varepsilon$ (in radians) and;

$$
C_{D}=C_{L} \varepsilon=\frac{C_{t}^{2}}{\pi \in A R}
$$

The geometry factor, e for all wing types elliptical, rectangular, swept back and trapezoidal is given by;

$$
\mathbf{e}=\frac{1}{1+\delta}
$$

For finite wing of general planform, the next equation (8) is the best describing the value of the lift slope (a). Of most importance in equation (8) is the aspect ratio variation. Note that for low (AR) wings, a substantial difference can exist between the lift slope of an infinite wing $\left(a_{0}\right)$ and the lift slope of a finite wing (a). The lift slope of the wing then can be determined as follows;

$$
\mathrm{dC}_{\mathrm{L}} / \mathrm{d} \alpha=\mathrm{a}=\frac{\mathrm{ao}}{1+\left(\frac{\mathrm{aD}}{\pi \mathrm{AR}}\right)(1+\tau)}
$$

Therefore, the lift coefficient of the wing is equal to the lift slope of the wing multiplied by the difference of the angles between the wing's angle of attack and the wing's zero lift angle of attack;

$$
\mathrm{C}_{\mathrm{L}}=\mathrm{a}\left(\propto-\propto_{\mathrm{L}=0}\right)
$$

The induced angle of attack for airfoil NACA 4412 can be calculated from;

$$
\propto_{i}=\frac{C_{L}}{\pi A R}
$$

As the effective angle of the wing is affected by the induced angle, then the effective angle will decrease by the calculated induced angle above to obtain;

$$
\propto_{\mathrm{eff}}=\propto-\propto_{\mathrm{i}}
$$

Finally, the lift coefficient of the airfoil is determined as follows;

$$
\mathrm{C}_{\mathrm{L}}=\mathrm{a}_{0}\left(\propto_{\mathrm{eff}}-\propto_{\mathrm{L}=0}\right)
$$

\subsection{Wing with Winglets Theoretical Work}

Fig.5 shows the various forces acting on the wing tip with winglet. Figure (a) shows a view in direction of flight. Figure (b) is the plan view, and Figure (c) shows the forces acting on winglets. Outboard of the tip, the flow is nearly circular as air from beneath the wing flows outward along the span, around the tip, and inward on the upper surface. The velocities induced by the wing are shown. To these the free-stream velocity is vectorially added. The magnitudes of the induced velocities generally increase toward the tip. At a given span wise location, the induced velocities are highest close to the surface of the wing, just outside the boundary layer (Mccormick, Barens W., 1995).

The severe limitations inherent in the assumptions leading to equation (14) below must be recognized (Mccormick, Barens $\mathrm{W}, 1995)$. For a given value of wing's wetted area to the whole wing's planform area $\left(\mathrm{S}_{\mathrm{w}} / \mathrm{S}\right)$, it would appear that increasing the reference area $\left(\mathrm{A}_{\mathrm{w}}\right)$ would always result in a smaller constant of proportionality, $\mathrm{K}$.

$$
\mathrm{C}_{\mathrm{D}}=\mathrm{C}_{\mathrm{d}}+\left[\mathrm{C}_{\mathrm{Di}}+\Delta \mathrm{C}_{\mathrm{Di}}\right]
$$

As $\mathrm{C}_{\mathrm{DW}} 0$ is the drag coefficient of the wing at zero lift, hence an increment in induced drag coefficient value $\Delta \mathrm{C}_{\mathrm{D} i}$ becomes;

$$
\Delta \mathrm{C}_{\mathrm{D}_{\mathrm{i}}}=-\frac{\mathrm{S}_{\mathrm{W}}}{\mathrm{S}}\left[2 \pi\left(\frac{\mathrm{A}_{\mathrm{W}}}{\mathrm{Aw}+2}\right)^{2} \mathrm{~K}^{2} \mathrm{C}_{\mathrm{L}}^{2}-\mathrm{C}_{\mathrm{D}_{\mathrm{W}_{0}}}\right]
$$

The approximate analysis by (Mccormick, Barens W.) indicates that:

1. The reduction in $C_{D}$ increases linearly with $C_{L}^{2}$.

2. At low $C_{L}$ values, $C_{D}$ will be increased by the addition of a winglet.

3. High winglet aspect ratio is desirable.

\section{Numerical Simulation}

The base line of our study is the theoretical solution. The governing aerodynamics equations are used to analyze the original wing parameters, then the modified design with winglets. We have selected $0^{\circ}$ and $5^{\circ}$ as the common angles of attack for the steady level flight or cruise flight. While $10^{\circ}$ angle of attack is the take-off angle of attack.

The numerical analysis was performed using two types of software: 3-D Foil-Multi surface code and Fluent ANSYS 14 code. Firstly, to analyze the original wing (Plain wing), then to analyze the wing with winglet, the iteration process is used to obtain converged solution.

\subsection{Computational Simulation}

The NACA airfoils are airfoil shapes for aircraft wings developed by the National Advisory Committee for Aeronautics (NACA). The shape of the NACA airfoils is described using a series of digits following the word "NACA". The parameters in the numerical code can be entered into equations to precisely generate the cross-section of the airfoil and calculate its 
specifications. The NACA four-digit wing sections define the profile by first digit describing maximum camber as percentage of the chord, second digit describing the distance of maximum camber from the airfoil leading edge in tens of percent's of the chord and last two digits describing maximum thickness of the airfoil as percent of the chord. The airfoil used in Jabiru aircraft, which we are studying in this work is NACA 4412, and the wing is rectangular with no aerodynamic twist and of constant chord and without winglets.

The main objectives of the plain wing winglets design are to:

- Decrease induced drag resulting in a total drag decrease.

- Reduce the strength of the tip vortex.

- Increase the lift to drag ratio.

- Decrease fuel consumption.

- Increase the flight range.

- Increase safety by eliminating the effect of wing tip vortices.

\subsection{Foil Multi-Surfaces Code (3-D)}

It is a simple version software which uses the thin airfoil theory model. Simplicity of this code allowed us to design the wing with winglet model. The suggested design needed around ten iterations to obtain the optimum design of the winglets. Then we proceeded to test and verify it numerically, and theoretically. The parameters changed in the design iteration using the 3-D Multi-Surface code are:

1. The span wise slope angle of the winglets to obtain an optimum angle at $\beta=65^{\circ}$.

2. The length of the winglets to be found optimum at $0.4 \mathrm{~m}$.

3. Fixed chord of the airfoils from tip to the root of the winglets.

4. NACA 4412 airfoil for the winglets root, and NACA 0012 for the winglets tip.

After obtaining the optimum design from the 3-D Foil Multi-Surfaces code, Solid Works is used to draw the wing with winglet final model ready to export to ANSYS Fluent 14 code.

\section{Theoretical Results}

The lift and drag coefficients results are calculated at $0^{\circ}, 5^{\circ}$, and $10^{\circ}$ angles of attack. The total drag is a combination of Parasite drag and induced drag. All the boundary conditions are measured at sea level, ambient temperature and Reynolds Number $R e=3.52 \mathrm{E}^{6}$ for free stream velocity of $50 \mathrm{~m} / \mathrm{s}$. Reynolds numbers are used in airfoil design to determine "Scale Effect" when computing flow characteristics. Aerodynamics Reynolds number, $R e, R e=V c / v$, where $V$ is the flow velocity, $c$ is the chord length, and $v$ is the kinematic viscosity of air surroundings the airfoil, which is $1.460 \times 10^{-5} \mathrm{~m}^{2} / \mathrm{s}$ for the air at sea level. By using 3-D Foil Multi-Surfaces and Solid Works codes, the final drawings for the two models plain wing and wing with winglet were obtained. For a constant airfoil NACA 4412, the plain wing is $9.528 \mathrm{~m}$ span and, and $1 \mathrm{~m}$ chord. While the wing with winglets as seen from top (reference area view) is $10.286 \mathrm{~m}$ span, $1 \mathrm{~m}$ chord, with the side angle of the winglets is $\beta=65^{\circ}$, with a constant airfoil NACA 4412, and the tip of the winglets airfoil is NACA 0012 , and the length of the winglet is $0.879 \mathrm{~m}$ for each side. Fig. 8 shows the top view of both models. Both wings are merged in one view to simplify the dimensions of the original and designed models. In the front view, the plain wing with 9.528 $\mathrm{m}$ span is seen. The winglets with the optimum side angle $\beta=65^{\circ}$ as determined from 3-D Foil Multi-Surface code was added, but with a different winglets tip airfoil. Both wings have a constant maximum camber through all the geometry of 0.12 m. Fig. 6 and Fig.7 show the top and front view of the plain wing with winglets.

\subsection{Theoretical Relations}

Table 1 shows the results of lift and drag coefficients versus angle $(\square)$ of attack for plain wing using the theoretical equations. $C_{L}$ stands for the lift coefficient of the 3-D wing, while $C_{d}$ presents the values of the profile drag, and $C_{D i}$ is the value of the induced drag for the wing. The summation of all drag values are listed under $\mathrm{C}_{\mathrm{D}}$. The calculated theoretical data are used as our base values, which are to be compared with the CFD simulation values afterwards. In addition, Table 2 shows the results of lift and drag coefficients versus angle of attack for the designed wing with winglets using the theoretical relations. All boundary conditions are exactly the same in both cases for the theoretical calculations.

\section{Numerical Results}

\subsection{3-D Foil Multi-Surfaces Code Results}

The results of this code for computed values of $C_{L}$ and $C_{D}$ are based on the thin airfoil theory. Results of Plain Wing are listed in Table 3, while results of the Wing with Winglets are listed in Table 4.

\subsection{Fluent ANSYS Code 14 Results}

The results of ANSYS 14 Code have shown very close resemblance to the theoretical results. Flow direction and vortices are monitored as well as the drag and lift coefficients results. The convergence of the solution is the sign for stable and précised results for both of drag and lift coefficients. The results for $C_{L}$ and $C_{D}$ for plain wing and that with winglets are shown in Tables 5 and 6 using the same boundary conditions and angles of attack. 


\section{Discussion of Results \\ 6.1 Induced Drag and Lift to Drag Ratios}

The comparison of the results between plain wing and the designed wing with winglets has shown that the induced drag has decreased with the plain wing with winglets in comparison to the plain wing model for all numerical methods as depicted in Fig.8 and Fig.9 at the various studied angles of attack. It is also noticed that the induced drag increases with angles of contact for all solutions. Using the Fluent code 14 has shown closer induced drag values to the mathematical (theoretical) solution for both plain and with winglets wing. The percentage reduction in induced drag between plain wing and wing with winglets results determined for all verification methods at the different angles of attack are; $8.5 \%$ at $\alpha=$ $0^{\circ}, 10 \%$ at $\alpha=5^{\circ}$, and $14.5 \%$ at $\alpha=10^{\circ}$ using the theoretical model, and 3\% at $\alpha=0^{\circ}, 4.4 \%$ at $\alpha=5^{\circ}$, and $6 \%$ at $\alpha=10^{\circ}$ using Fluent Ansys 14 simulation code. Moreover, the percentage reduction simulated using the 3-D Multi-Surfaces code is $13 \%$ at $\alpha=0^{\circ}, 8 \%$ at $\alpha=5^{\circ}$, and $7.7 \%$ at $\alpha=10^{\circ}$. The designed wing with winglets improved the aerodynamic characteristics of the wing by decreasing the induced drag values at different angles of attack.

The second main objective of this study is to show that the winglets design has a significant effect in improving the lift to drag ratio. This ratio has increased especially at high angles of attack due to the reduction of the amount of the induced drag, which allow the total drag coefficient to decrease and the lift coefficient to increase at the same time. Different numerical results obtained from each working method for plain wing are shown in Fig.10 and for the designed wing with winglets in Fig.11. After processing the solution of the designed wing with winglets in Fluent Ansys 14, it is shown that the flow characteristics of the wing will be improved after applying the winglets with the optimum winglet's geometry. The vortices are shifted to the edge of the winglet and this shift moves all the vortices to the winglets instead of the wing tips. Because of this effect, the stability and maneuverability increase at high angles of attack. The Induced drag decreases due to that as well as the lift to drag ratio increases. The tip vortices in this case will not spoil the lift on the wing tips and the resistance will decrease. 6.2 Plain wing and wing with winglets pressure comparison

The total pressure of the wing is a major factor in all aerodynamics studies. The airfoil shape of the wing's cross section divides the pressure into two separate zones: The lower surface of the wing is a high pressure zone, and the upper surface of the wing is a low pressure zone. The difference in the pressure between these zones creates the lift. In the chord's direction (the 2-D direction) it is noticed that the major effect of the winglets is smoothing the pressure gradient on both airfoil surfaces. As seen in Fig.12, the lower surface of the plain wing airfoil has significant fluctuations increased at the leading and trailing edges as depicted from the ANSYS 14 code simulation. In Fig.13, for wing with winglets both airfoil surfaces show stabilization in the pressures. This stabilization will allow getting the maximum benefit of the pressure difference to generate the lift. This phenomenon is more noticeable at the upper airfoil surface. When the total pressure is observed for the plain wing in 3-D view as seen from frontal view in Fig. 14, the pressure distribution in wing span direction is almost constant from the tip to tip for the plain wing. The 3-D shape of the plain wing shows approximately $1.7 \mathrm{MPa}$ difference between the upper and lower wing's surfaces. While in Fig.15, the pressure difference between upper and lower surfaces for the wing with winglets is approximately $2 \mathrm{MPa}$. This increment in pressure difference means that the lift force generated by the wing in the case of wing with winglets is bigger and will result in increasing the lift to drag ratio.

The difference in total pressure at the wing's tips for the wing with winglets is approximately

$5 \mathrm{MPa}$, but in the plain wing wing's tips is only $2.5 \mathrm{MPa}$. This can be explained by the effect of the winglets as they decrease the wing tip vortices from circulation around the wing's tip. This phenomenon generates high pressure difference between upper and lower tips. This high pressure difference increases the stability of the wing as a new benefit from the winglets design.

\section{Conclusions}

Theoretical and numerical work have been performed to examine the efficiency of winglets mounted on an airfoil in subsonic flows. The winglets that are considered here are placed upward at the tip of the wing in order to improve the wing efficiency; decreasing the induced drag and increasing the lift to drag ratio.

ANSYS CFD simulation shows that mounting a simple winglet at the tip of the aircraft wing can reduce the induced drag significantly that is always desired. Though this theoretical concept was established by the flow simulation over the same model aircraft wing when one of them was equipped with a winglet at the wing tip and another one is not. Results obtained from flow simulation describe that, if angle of attack, thermodynamics and other parameters are being kept constant then a conventional aircraft wing having winglet will significantly generate less induced drag compared to other conventional aircraft wing having no winglet. This can be considered as a great achievement from an aerodynamic point of view.

The main results achieved in this study can be described in the following points:

1.The theoretical work shows that decrement in the values of induced drag achieved for our designed wing with winglets is $8.5 \%$ at $0^{\circ}$ angle of attack, $10 \%$ at $5^{\circ}$ angle of attack, and $14.5 \%$ at $10^{\circ}$ angle of attack. The values of the lift to drag ratio increased by $15 \%$ at $0^{\circ}$ angle of attack, $7 \%$ at $5^{\circ}$ angle of attack, and $13.3 \%$ at $10^{\circ}$ angle of attack.

2.The numerical work was performed using two different codes. First, Fluent Ansys 14 is used to verify the results by the most realistic values in simulating the plain wing and wing with winglets. The values of induced drag for the designed wing with winglets decreased $3 \%$ at $0 \square$,

$4.4 \%$ at $5^{\circ}$, and decreased by $6 \%$ at $10^{\circ}$ angle of attack. The lift to drag ratio increased by $9.5 \%$ at $0^{\circ}$ angle of attack and increased by $4.4 \%$ at $5^{\circ}$ angle of attack, and increased by $18.7 \%$ at $10^{\circ}$ angle of attack.

3.The second numerical verification method used is 3-D Foil Multi-Surfaces Code. The values of the induced drag decreased $13 \%$ at $0^{\circ}$ angle of attack, $8 \%$ at $5^{\circ}$ angle of attack and $7.7 \%$ at $10^{\circ}$ angle of attack. The lift to drag ratio at $0^{\circ}$ angle of attack increased by $8.5 \%, 16 \%$ at $5^{\circ}$ angle of attack and $22 \%$ at $10^{\circ}$ angle of attack. 
4.The stability and maneuverability improved in our designed wing with winglets. This is noticeable at high angles of attack at low speeds. This presents the condition in take-off, where the induced drag contributes by nearly $60 \%$ of the total drag ratio (Jones, R., 1990). In our study the induced drag approached $70 \%$ of the total drag value. This benefit emphasizes the safety required for this type of aircraft under study.

5.As a result of decreasing the induced drag and increasing the lift to drag ratio, this will add an increment in the range, which will add a benefit to the ALS aircraft which is mainly owned by its users because it is an economical aircraft.

6.There are some differences between the theoretical and numerical methods used in the verification work due to several reasons, namely, the governing equations used by each numerical method is different due to the designer's code concept and also the selected boundary conditions.

\section{References}

[1] Bento Mattos, Antonini Macedo, and Durval Silva Filho (2003). Consideration about winglet design, proceedings 21st Applied Aerodynamics Conference, Florida, U.S.A.

[2] I. Kroo L (2005), Nonplanar wing concepts for increased aircraft efficiency. VKI lecture series on innovative configurations and advanced concepts for future civil aircraft, pp.1-29.

[3] Hantrains-Gervois et al. (2009), Effect of gurney flaps and winglets of the performance of HAWT, chapter 6.

[4] Ning A., Kroo L (2010), Multi-disciplinary considerations in the design of wings and wing tip devices, Journal of aircraft, vol.47, pp.534-543.

[5] Jansen, Perez, and Ra Martins (2010), A python-based object-oriented frame work for non-linear constrained optimization, Journal of structural and multi-disciplinary optimization,vol.45, issue 1, pp.101-118.

[6] Nurulain, Y., Jamaluddin (2012), S. Flow behavior around winglets. Journal Mechanical, vol.34, pp. 95-100.

[7] Gimmestad, (1981). Technology reshaping aviation partners winglets, Aviation partners, internet website.

[8] Azlin M.A., Maltaib C.F., Kasolang S. (2010), and Muhammed F.H. CFD analysis of winglets at low subsonic flow, proceedings of the world congress on engineering, WCE, vol.1, pp.87-91.

[9] Takenaka K., Hatanaka, K., Yamazaki W., and Nakahashi K. (2008), Multidisciplinary design exploration for a winglet, Journal of aircraft, vol 45, 5, pp.1601-1611 .

[10] Anderson, J. D. (2003), Fundamentals of Aerodynamics (4th ed.). New York: McGraw-Hill .

[11] McCormick, Barens W. (1995), Aerodynamics Aeronautics and Flight Mechanics (2nd ed.). John Wiley \& SONS, INC.

[12] Jones, R. Wing Theory (1990). Princeton, NJ: Princeton University Press.

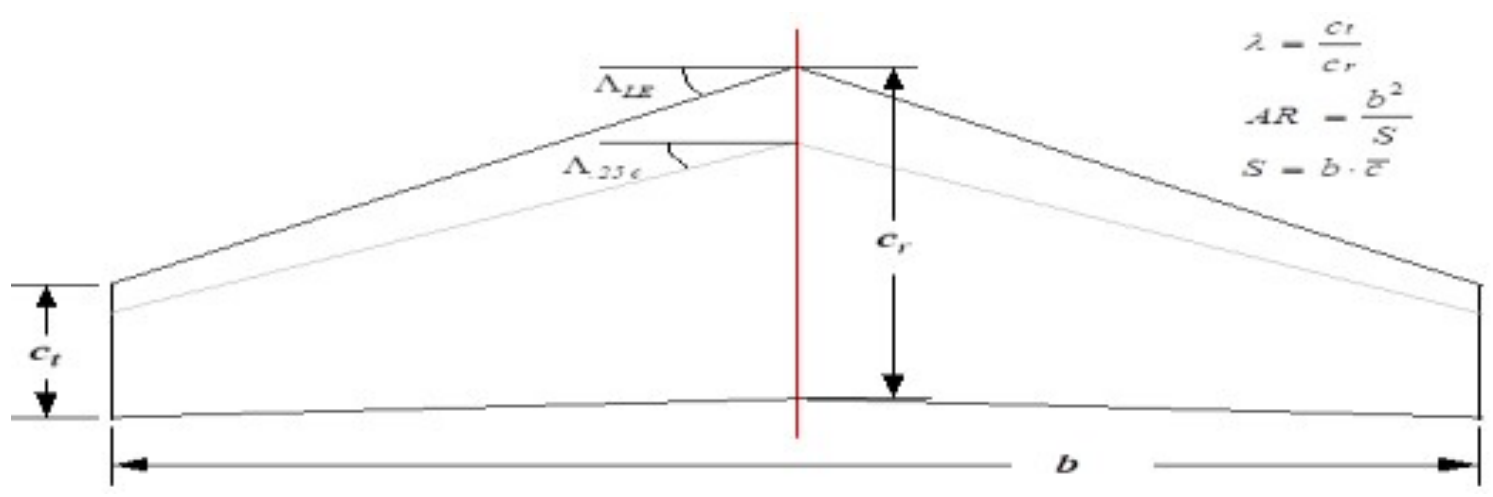

Fig.1 Finite wing geometry definitions

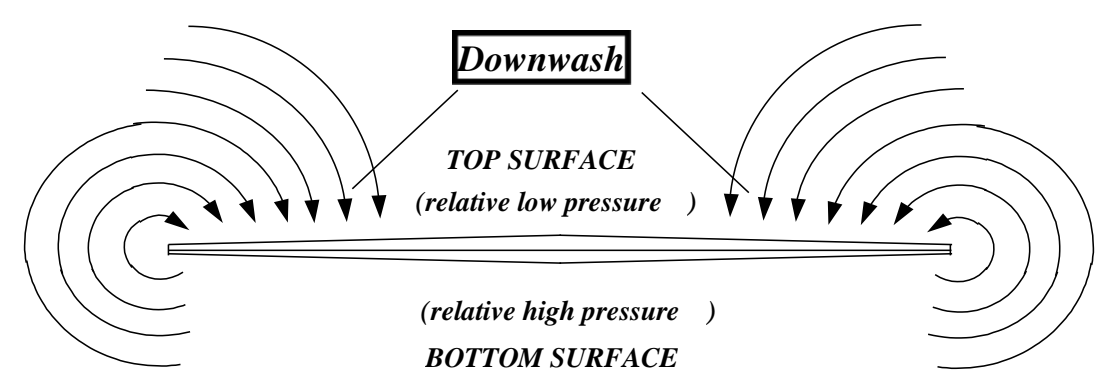

Fig.2 Front view of wing with flow around the wing tips 


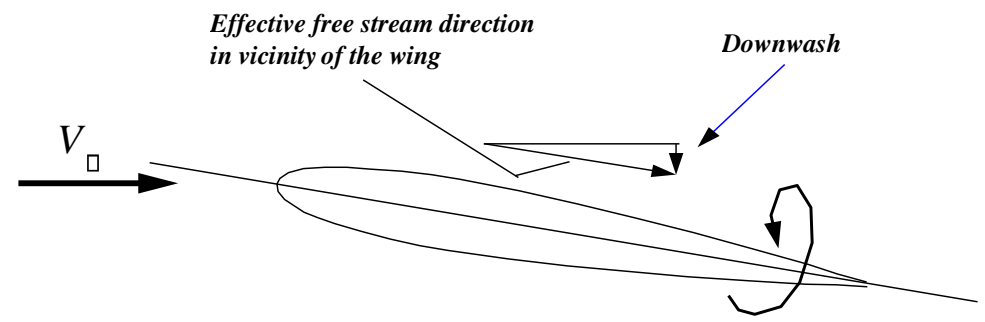

Fig.3 Downward flow component - Downwash

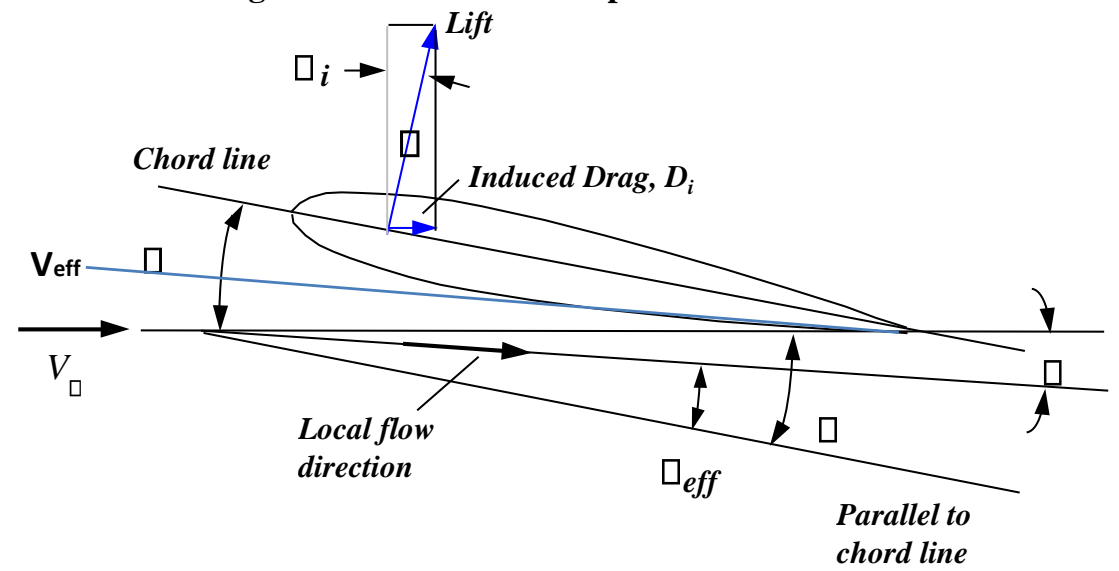

Fig.4 Induced drag definition

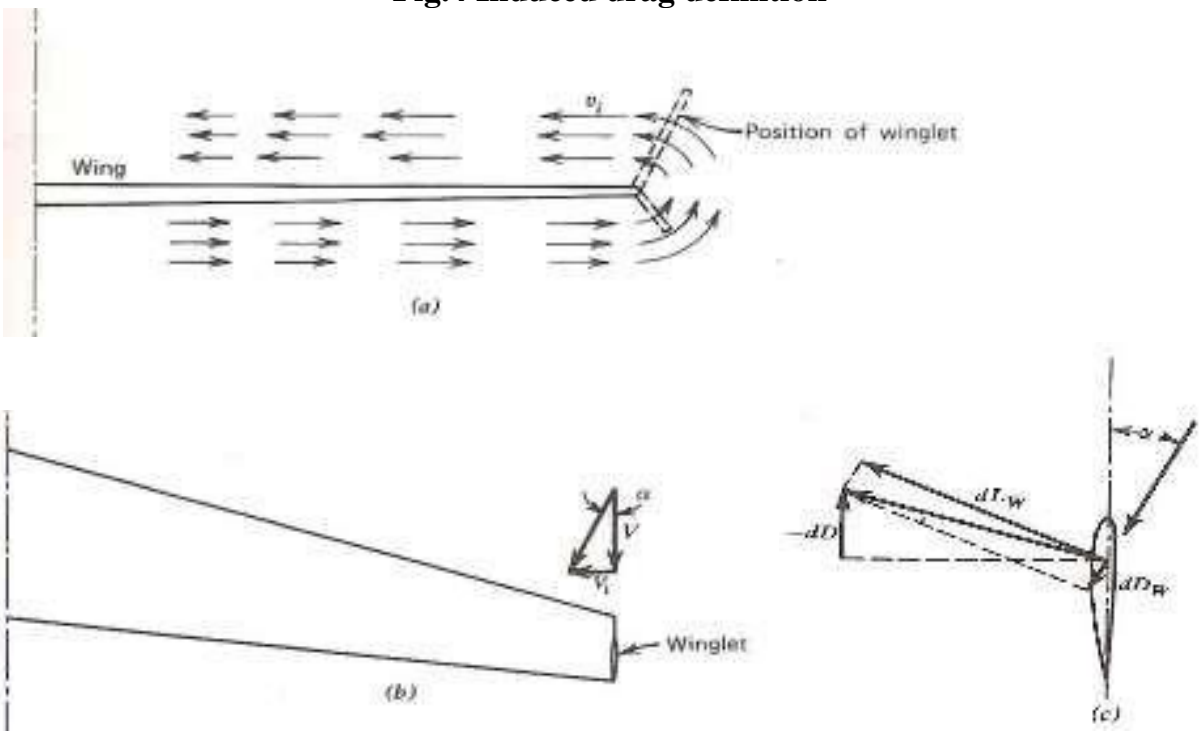

Fig.5 Generation of negative drag by winglets (a) Direction of flight (b) Plan view (c) Forces acting on winglet (Source: Mccormick, Barens W, 1995)
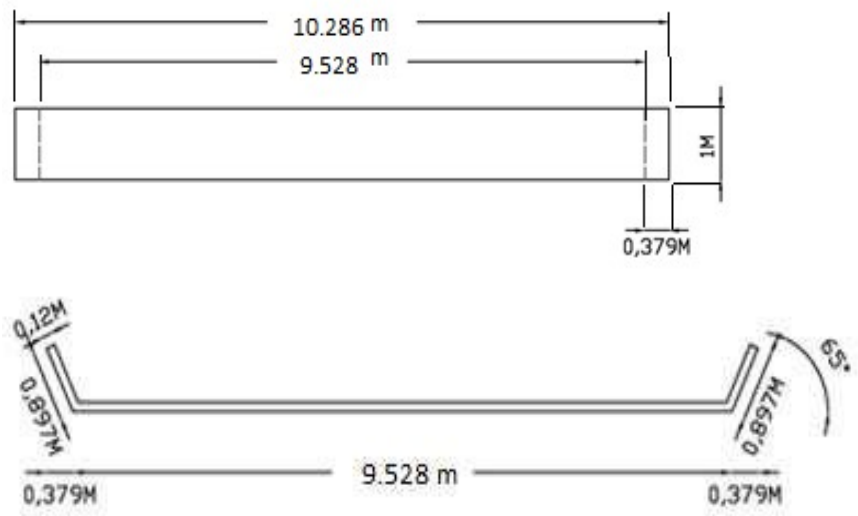

Fig.6 Top view for the plain wing with winglets Fig.7 Front view for the plain wing with winglets 
Table 1 Lift $C_{L}$ and drag $C_{d}$ coefficients versus angle of attack $(\alpha)$ for plain wing using theoretical relations

\begin{tabular}{|c|c|c|c|c|}
\hline$\alpha$ & $\mathrm{C}_{\mathrm{L}}$ & $\mathrm{C}_{\mathrm{d}}$ & $\begin{array}{c}\text { Induced drag } \\
\left(\mathrm{C}_{\mathrm{Di}}\right)\end{array}$ & Total drag $\left(\mathrm{C}_{\mathrm{D}}\right)$ \\
\hline $0^{0}$ & 0.246 & 0.0076 & 0.0047 & 0.0123 \\
\hline $5^{0}$ & 0.863 & 0.0112 & 0.0275 & 0.0387 \\
\hline $10^{0}$ & 1.368 & 0.0203 & 0.069 & 0.0894 \\
\hline
\end{tabular}

Table 2 Results of lift and drag coefficients versus angle of attack for the designed wing with winglets

\begin{tabular}{|c|c|c|c|c|}
\hline$\alpha$ & $\mathrm{C}_{\mathrm{L}}$ & $\mathrm{C}_{\mathrm{d}}$ & $\begin{array}{c}\text { Induced drag } \\
\left(\mathrm{C}_{\mathrm{Di}}\right)\end{array}$ & $\begin{array}{c}\text { Total drag } \\
\left(\mathrm{C}_{\mathrm{D}}\right)\end{array}$ \\
\hline $0^{0}$ & 0.276 & 0.0077 & 0.0043 & 0.0120 \\
\hline $5^{0}$ & 0.8800 & 0.0115 & 0.0247 & 0.0362 \\
\hline $10^{0}$ & 1.395 & 0.0238 & 0.0590 & 0.0828 \\
\hline
\end{tabular}

Table 3 Lift and drag coefficients versus angle of attack for plain wing using 3-D Foil Multi-Surfaces Code

\begin{tabular}{|c|c|c|l|c|}
\hline$\alpha$ & $\mathrm{C}_{\mathrm{L}}$ & $\mathrm{C}_{\mathrm{d}}$ & $\begin{array}{c}\text { Induced drag } \\
\left(\mathrm{C}_{\mathrm{Di}}\right)\end{array}$ & $\begin{array}{c}\text { Total drag } \\
\left(\mathrm{C}_{\mathrm{D}}\right)\end{array}$ \\
\hline $0^{0}$ & 0.2254 & 0.0075 & 0.0023 & 0.0098 \\
\hline $5^{0}$ & 0.5667 & 0.0073 & 0.0154 & 0.0227 \\
\hline $10^{0}$ & 0.90 & 0.0110 & 0.039 & 0.050 \\
\hline
\end{tabular}

Table 4 Lift and drag coefficients versus angle of attack for wing with winglets using 3D Foil Multi-Surfaces Code

\begin{tabular}{|c|c|c|c|c|}
\hline$\alpha$ & $\mathrm{C}_{\mathrm{L}}$ & $\mathrm{C}_{\mathrm{d}}$ & $\begin{array}{l}\text { Induced drag } \\
\left(\mathrm{C}_{\mathrm{Di}}\right)\end{array}$ & $\begin{array}{c}\text { Total drag } \\
\left(\mathrm{C}_{\mathrm{D}}\right)\end{array}$ \\
\hline $0^{0}$ & 0.246 & 0.0075 & 0.0020 & 0.00945 \\
\hline $5^{0}$ & 0.642 & 0.0073 & 0.0141 & 0.0214 \\
\hline $10^{0}$ & 1.053 & 0.011 & 0.0360 & 0.047 \\
\hline
\end{tabular}

Table 5 Lift and drag coefficients versus angle of attack for plain wing using Fluent ANSYS 14 Code

\begin{tabular}{|c|c|c|c|c|}
\hline$\alpha$ & $\mathrm{C}_{\mathrm{L}}$ & $\mathrm{C}_{\mathrm{d}}$ & $\begin{array}{c}\text { Induced } \\
\left(\mathrm{C}_{\mathrm{Di}}\right)\end{array}$ & 0.0144 \\
\hline $0^{0}$ & 0.302 & 0.0075 & 0.0069 & 0.0329 \\
\hline $5^{0}$ & 0.768 & 0.0100 & 0.0229 & 0.072 \\
\hline $10^{0}$ & 1.164 & 0.0240 & 0.048 & Total drag $\left(\mathrm{C}_{\mathrm{D}}\right)$ \\
\hline
\end{tabular}

Table 6 Lift and drag coefficients versus angle of attack for wing with winglets using Fluent 14 Code

\begin{tabular}{|c|c|c|c|c|}
\hline$\alpha$ & $\mathrm{C}_{\mathrm{L}}$ & $\mathrm{C}_{\mathrm{d}}$ & Induced $\left(\mathrm{C}_{\mathrm{Di}}\right) \mathrm{drag}$ & Total drag $\left(\mathrm{C}_{\mathrm{D}}\right)$ \\
\hline $0^{0}$ & 0.34 & 0.0075 & 0.0067 & 0.0142 \\
\hline $5^{0}$ & 0.8 & 0.0101 & 0.0219 & 0.032 \\
\hline $10^{0}$ & 1.25 & 0.0180 & 0.0450 & 0.063 \\
\hline
\end{tabular}




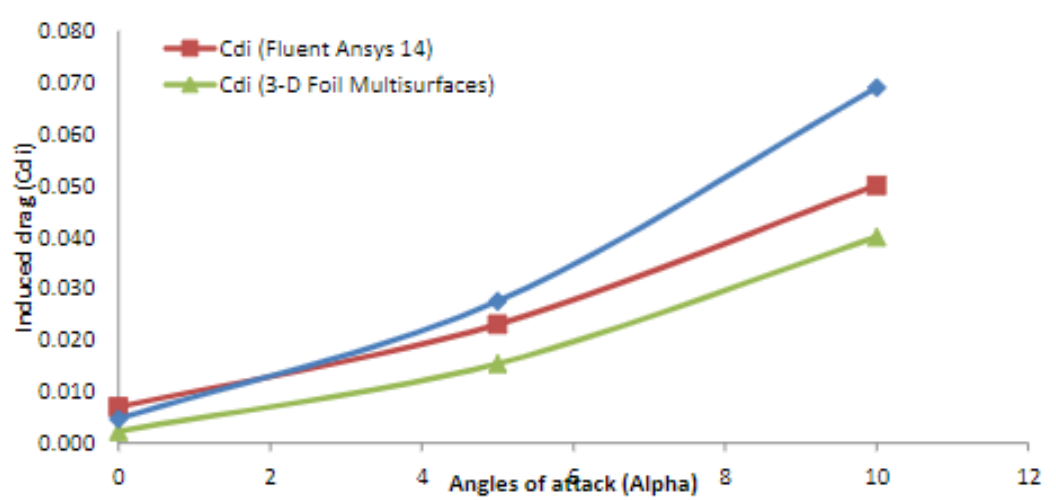

Fig.8 Induced drag $\left(\mathrm{C}_{\mathrm{Di}}\right)$ for plain wing at different angles of attack $(\alpha)$

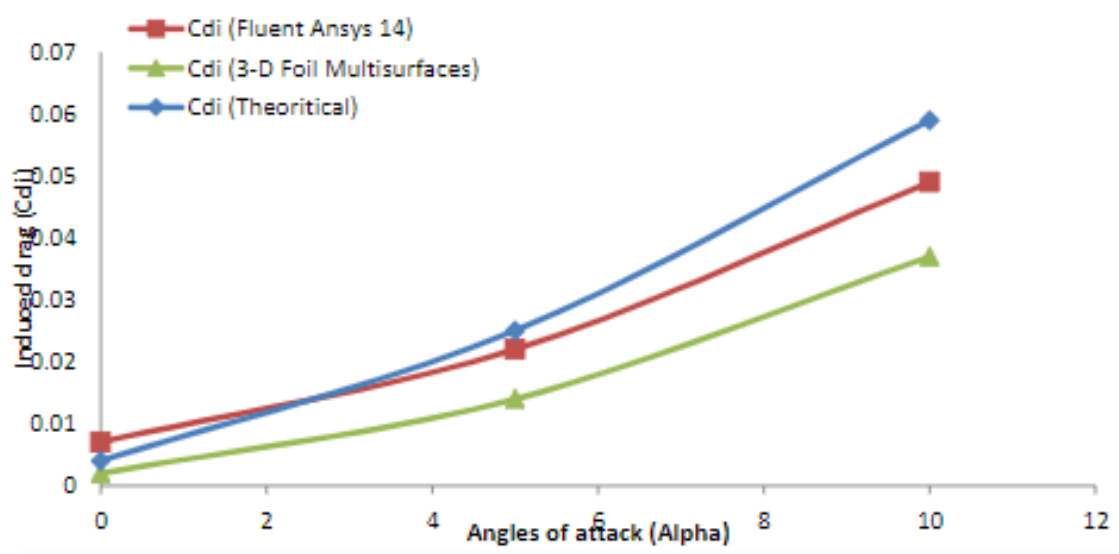

Fig.9 Induced drag $\left(\mathrm{C}_{\mathrm{Di}}\right)$ for wing with winglets at different angles of attack $(\alpha)$

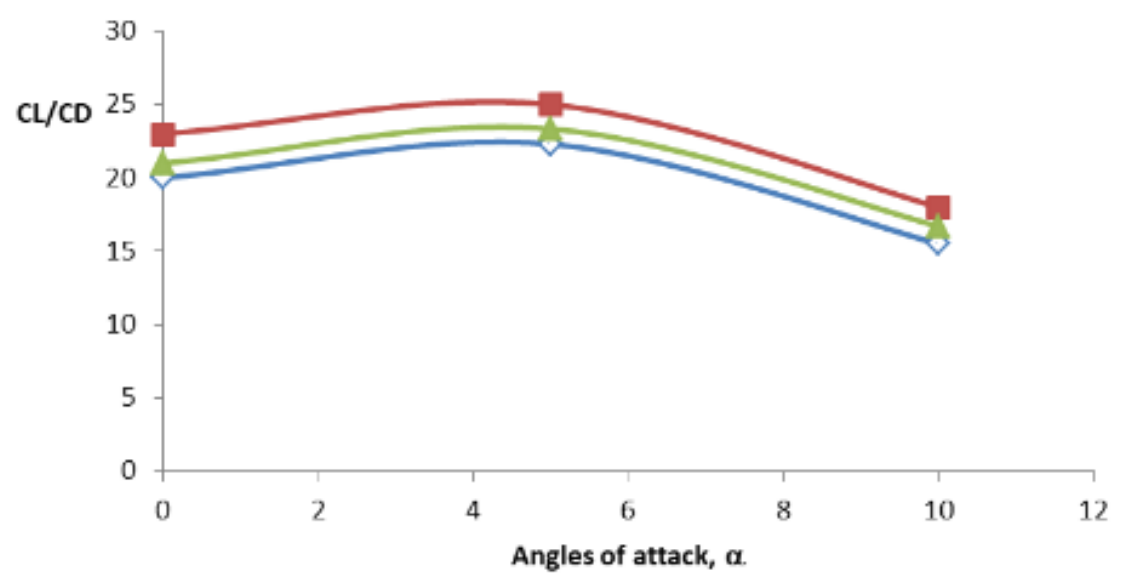

Fig.10 Lift to drag ratio $\left(C_{L} / C_{D}\right)$ comparison for plain wing at different angles of attack

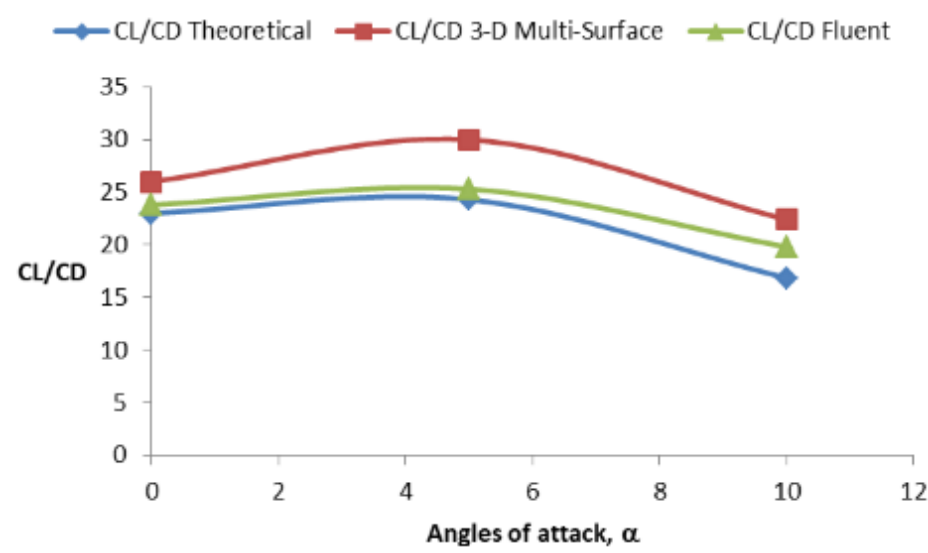

Fig.11 Lift to drag ratio $\left(C_{L} / C_{D}\right)$ comparison for wing with winglets at different angles of attack 


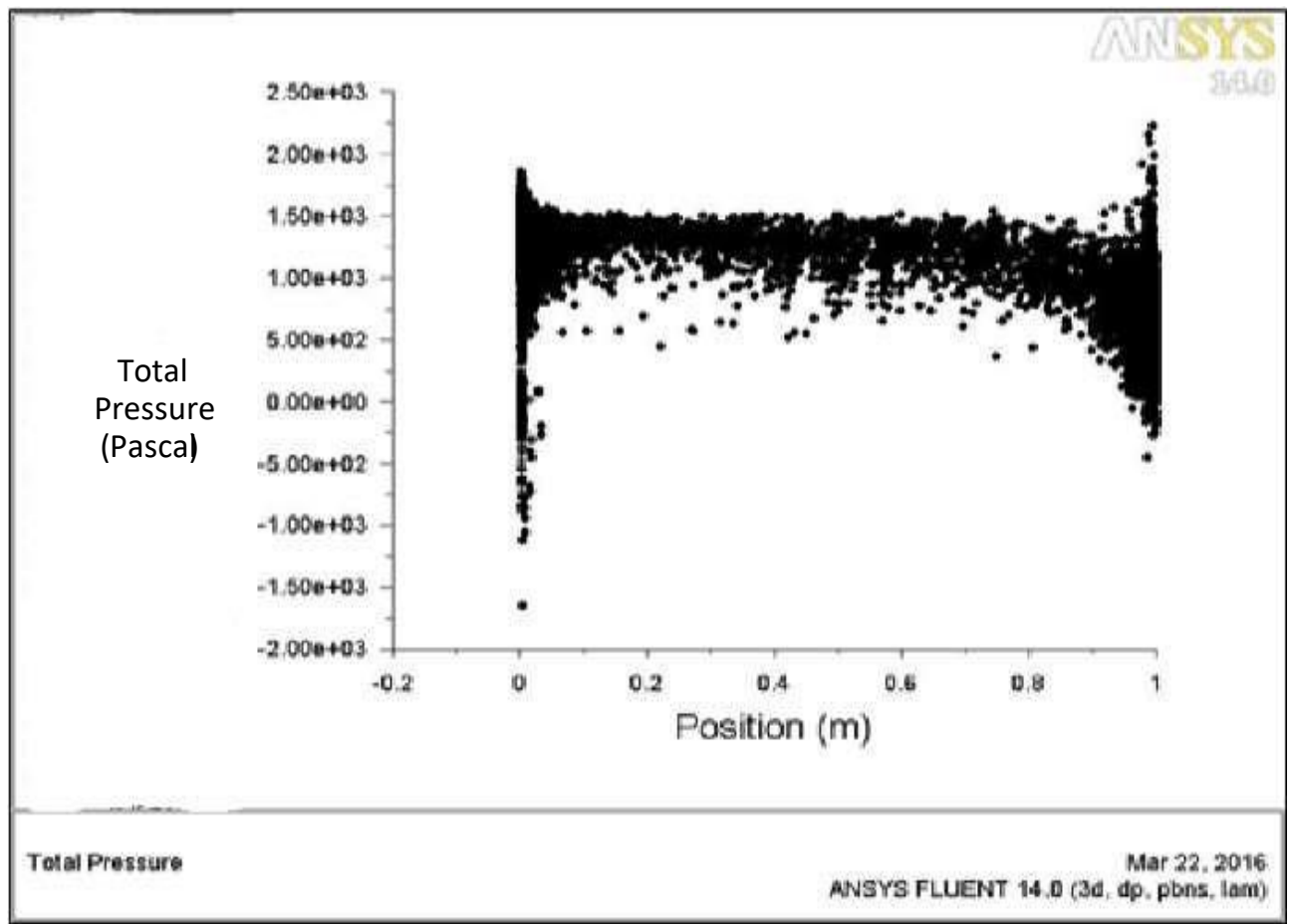

Fig.12 Plain wing chord's direction total pressure

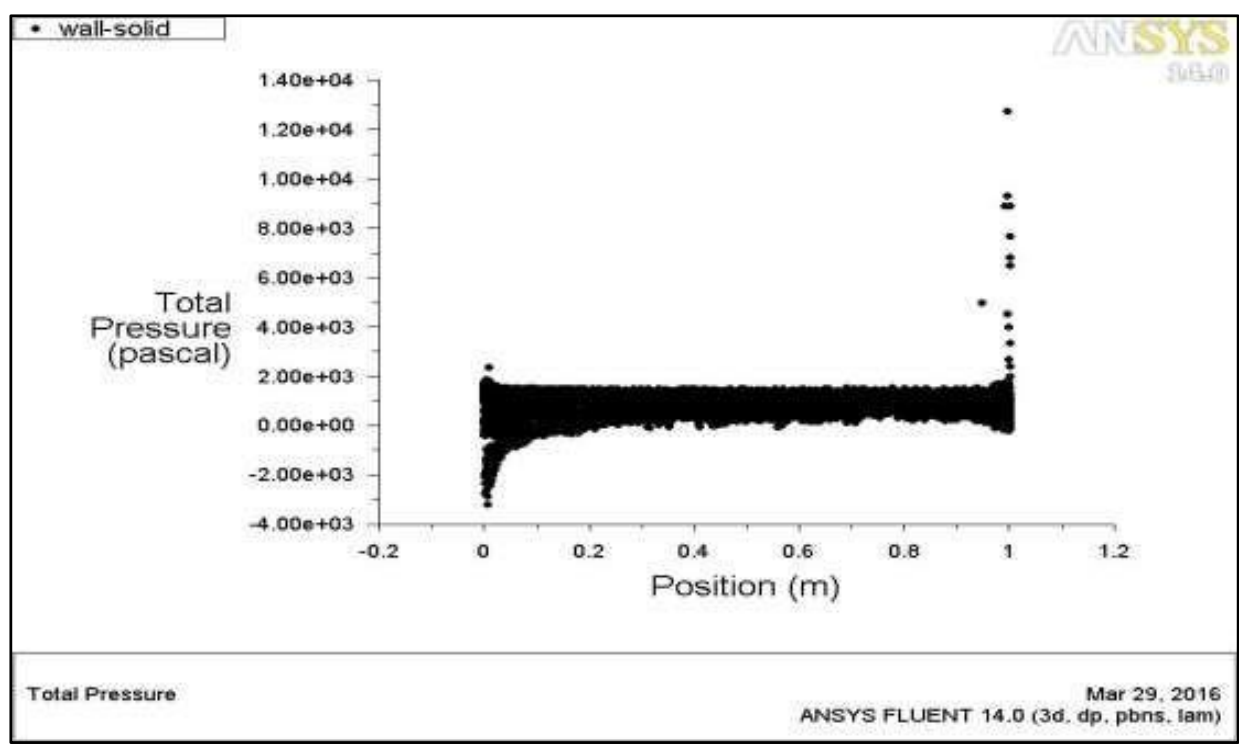

Fig.13 Wing with winglets chord's direction total pressure

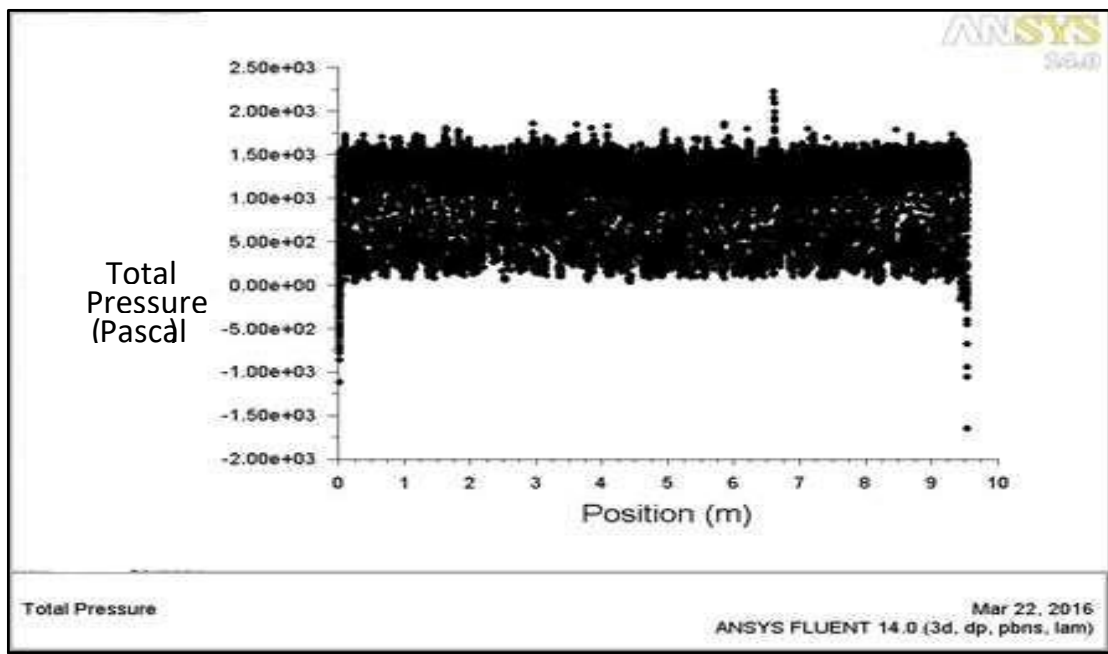

Fig.14 Plain wing span direction total pressure 


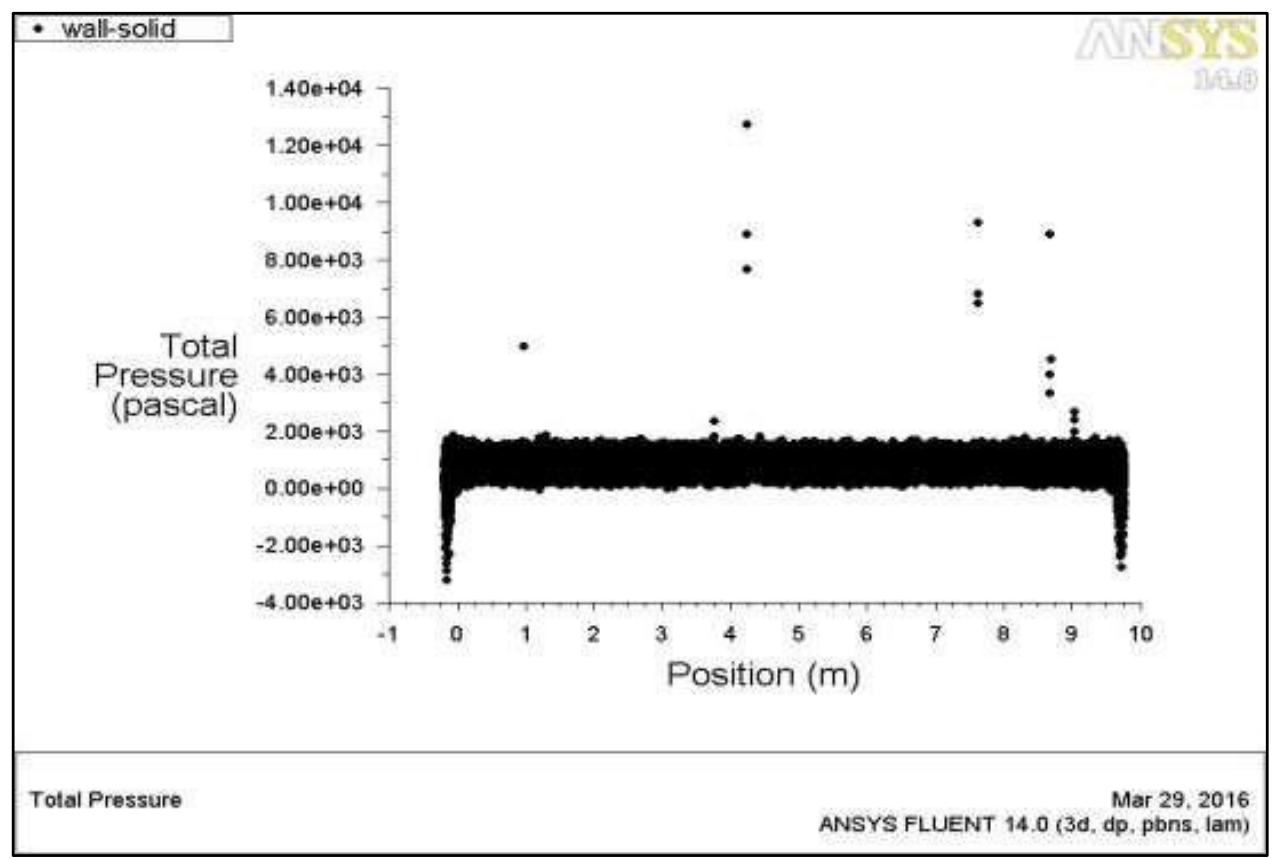

Fig.15 Wing with winglets in wing span direction total pressure 